\title{
Comparative Analysis of Single Image Shadow Detection and Removal in Aerial Images
}

\author{
Abhishek Mishra, Bharti Chaurasia and Yashwant Kurmi
}

\begin{abstract}
The article for the specific region segmentation is discussed here. This paper compares the work done on the contour model based shadow detection algorithm with the random field model based shadow detection method. The contouring based shadow detection model adapts the traditional geometric active contours that the contour is steadily subjective toward the shaded region and the shadowy regions in the picture. The shadow region segmentation follows the post processing for optimal threshold calculation. The complexity metric is constructed to separate the boundary of true shadow regions. Whereas the random field model based on shadow exposure process uses a superficial outside descriptor, and colour shade area, to describe the colour surface variants. The conditional random field is combined with the colour descriptors to locate lighting pair and acquire the shaded portion. The shadow region riddance by neighbourhood shade constancy utilizes anisotropic dispersion to guess the local image pixel illumination in shadow region and provides the better performance in shadow region illumination enhancement.
\end{abstract}

\section{Keywords}

Shadow detection; Shadow removal; Aerial images; Conditional random field, Contour detection

\section{INTRODUCTION}

The shadow regions are the area created by obscuring light source through an object. The regions defined as shadow can either aid or confuse scene understanding, subject to whether it models the shadow or ignore it. If the shadows are detected and removed it can better localize objects and understand its shape. It helps to determine the objects borders and contact with the ground as shown in fig. 1.

Recently the active contour based method [1] and the conditional random field based shadow detection method [2] performed a significant task in the field of shadow detection. The monochromatic images [3] and the color images are considered in these shadow detection tasks. The identified shadow regions also offer many signal for illumination surroundings [4], and all the nearby sight geometry [5]. If the shadow regions are ignored, the forged edges on the boundary of the shadowed surfaces and mystification between albedo and shadow can direct to inaccuracy in pictorial representation. The above mentioned reasons have been measured a critical section of picture analysis (e.g., [6], [7]). The basic image processing [8-13] and the formulation of the shadow detection background [14] is discussed in the further part of the paper.
In this paper, the shadow detection and removal techniques are analysed and compared. The second part of this paper has the shadow region segmentation based on geometric feature based active contouring. The third section provides the shadow detection and removal based on conditional random field model. The fourth section provides the detail of the result analysis. The final fifth section concludes the paper with the future scope in the field of shadow detection.

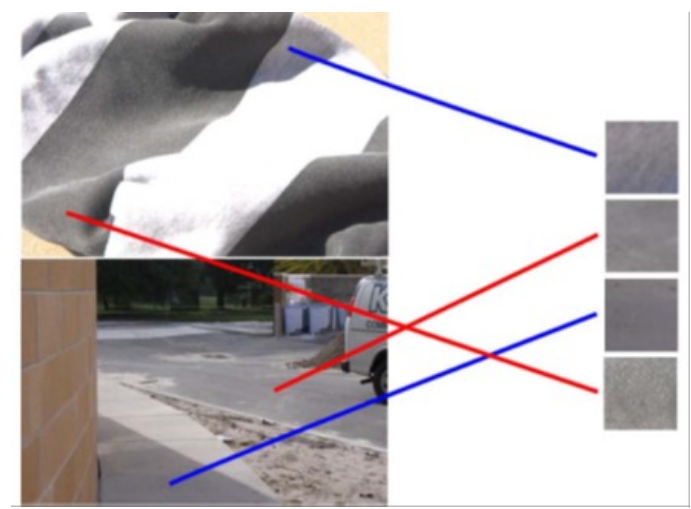

Fig. 1. The local region appearance with ambiguous regions; to find shadows, it compares the surfaces of the same material [18].

\section{ACTIVE CONTOURING}

The region segmentation for the clear border extraction in the image is done by the contouring through active edges. The geometry based active contour [1] is applied in the energy equation to bias the contours in the direction of the borderline for the extraction of dark and shadow regions. The active contouring provides direction to determine the radiometric appearances of dark areas corresponding to neighbours. The outcome of the active contouring is considered as a cue for presence of shadows region.

\section{A. Level-Set Formulation}

The suitable energy is computed and stored for the further processing by the suitable set selection. The criteria for the particular set selection are discussed in the following equation (1). The level-set method $[15,16]$ employed to compute energy function over the input image considers the domain $\Omega$. The method describes the curve $\mathrm{C}$ which is characterized by zero level set of the function: $\Omega \rightarrow \mathrm{R}+$, as

$$
\left\{\begin{array}{l}
C=\{(x, y) \in \Omega: \psi(x, y)=0\} \\
\text { inside }(C)=\{(x, y) \in \Omega: \psi(x, y)>0\} \\
\text { outside }(C)=\{(x, y) \in \Omega: \psi(x, y)<0\}
\end{array}\right.
$$


The curve $\mathrm{C}$ is then replaced by function $\psi$. The additional function Heaviside $\mathrm{H}$ is used in it for the simplification of the model. The 1-D Dirac measure $\delta$ is also provides the simplicity and is given as follows:

$$
\begin{gathered}
H(\psi)= \begin{cases}1, & \text { if } \psi \geq 0 \\
0, & \text { if } \psi<0\end{cases} \\
\delta(\psi)=\frac{d}{d \psi} H(\psi)
\end{gathered}
$$

The Heaviside $H$ and Dirac $\delta$ functions, are related by the mathematical relation as here Dirac $\delta$ functions is a derivative of $\mathrm{H}$, and is approximated by the following functions:

$$
\begin{aligned}
& H_{\epsilon}(z)= \begin{cases}1, & \text { if } z>\epsilon \\
0, & \text { if } z<-\epsilon \\
\frac{1}{2}\left[1+\frac{z}{\epsilon}+\frac{1}{\pi} \sin \left(\frac{\pi z}{\epsilon}\right)\right], \text { if } z \leq \epsilon\end{cases} \\
& \delta(z)_{\epsilon}=\left\{\begin{array}{l}
0, \quad \text { if }|z|>\epsilon \\
\frac{1}{2 \epsilon}+\frac{1}{2 \in} \cos \left(\frac{\pi z}{\epsilon}\right), \quad \text { if }|z| \leq \epsilon
\end{array}\right.
\end{aligned}
$$

The level set selection provides the energy region wise particularly. The sectional energy is computed and stored for further processing. There may be some other problem of the noisy or clutter regions. The clutter region can also be separated from the shadow region by the following process in the next subsection.

\section{B. Clutter Separation from Shadow}

The regions have the low intensity (or the dark regions) found by contouring model for the geometry based active contours include shadow and clutter as much separable. It employs the following procedural steps to remove the clutter. The result of the method is a set of probable shadow regions $\mathrm{R}$ $=\{\mathrm{R} 0, \mathrm{R} 1, \ldots, \mathrm{Rn}-1\}$ that may comprise clutter. The clutter removal from the probable shadow regions, it processes further the result as follows. The construction of Otsu's threshold on global histogram is done for the detected regions after adding a strip to each region, from the surrounding. The Otsu's threshold method provides an optimal threshold to segment a bimodal histogram automatically. The experimental result shows that the three pixels strip from the surrounding region for each region are adequate to obtain a global bimodal histogram for the detected regions. The bimodal histogram illustrates that; one peak is for the shadow and the pixels of similar intensity in segmented regions. The other peak illustrates the surroundings' pixels and pixels of similar intensity. The clutter removal form the shadow region by the global Otsu's threshold in which the clutter region falls within the peak of the surrounding pixels. And also increases the complexity and irregularity of boundaries of the remaining clutter that fall between the two peaks of the bimodal histogram [1]. The geometric filter with higher boundary conditions helps in removing the clutter.

The algorithm discussed here is for detection and segmentation of shadow of man-made buildings in panchromatic satellite images. The algorithm is systematically biased and fully automated for shadow detection. It removes the clutter regions associated with shadow regions by using an integrated optimal segmentation methodology with geometric filter to separate the clutter from the shadow in images.

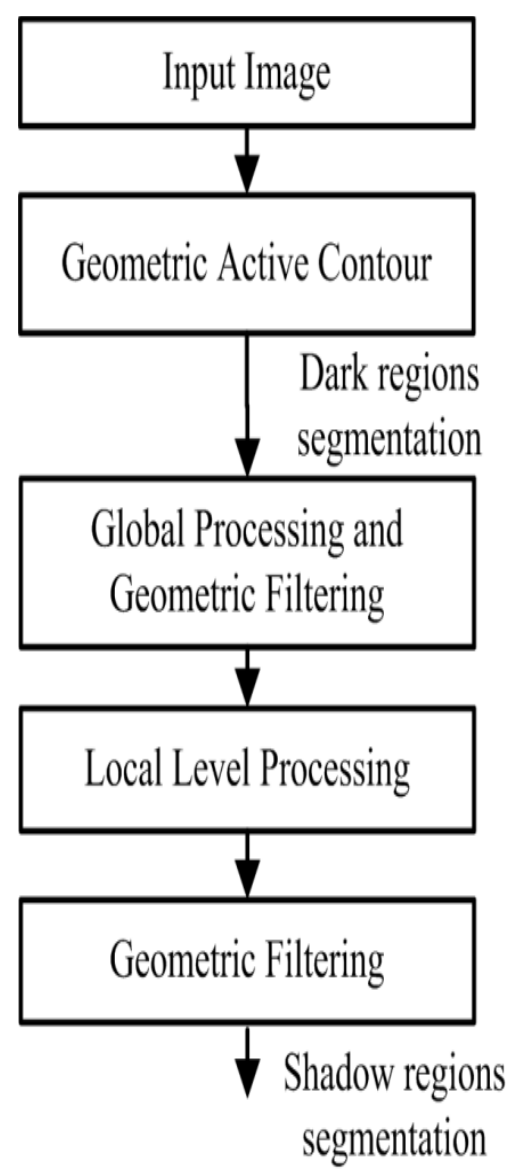

Fig. 2. Flowchart of the contour based shadow detection method.

\section{CONDITION RANDOM FIELD MODEL}

The conditional random field model has the parallel processing in the map extraction and the AM/LM correlation. The flowchart of the conditional random field based method is shown in figure 3 with clear summarized illustration of the processing steps.

\section{A. Anisotropic local colour constancy}

In the shadow region detection by the CRF model [2], the shadow and non-shadow regions are distinguished by the illumination intensity, colour and texture appearance. The image segmentation by conditional random field (CRF) based method uses the mean shift algorithm [17]. Let us assume that the numbers of segmented regions are $\mathrm{n}$ in the image. The shadow region is detected by constructing a graph $G=(V, 1)$ with edges $\varepsilon$ and vertices $V$ that represents the image. 


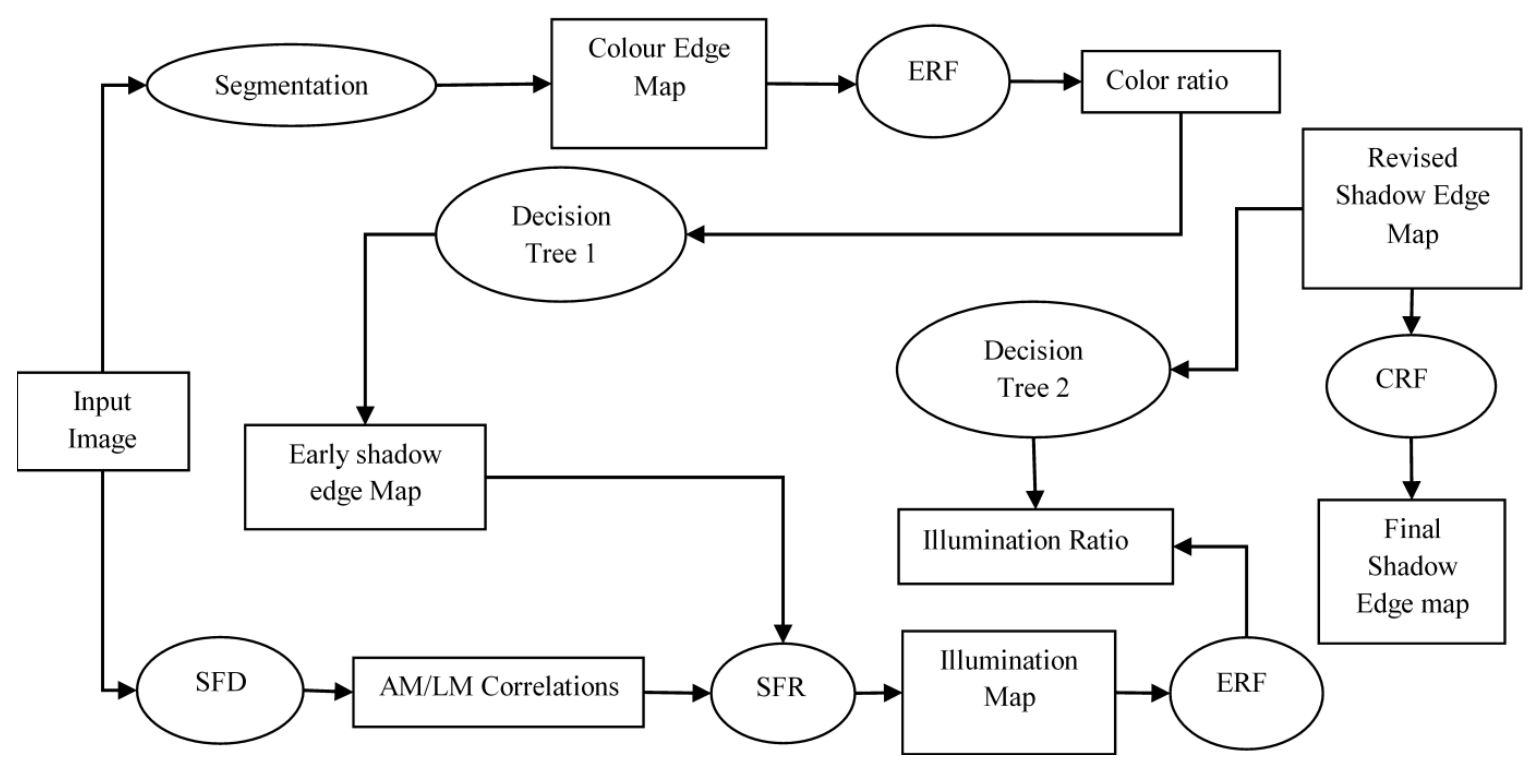

Fig. 3. Flow chart of conditional random field (CRF) based shadow detection method [2].

The edges set $\varepsilon$ represents various illumination pairs. It defines a conditional random field (CRF) on that graph, that expresses the log-likelihood of a particular labelling of the given observed data $x i$ in terms of a sum of unary potential $S\left(x_{i}, y_{i}\right)$ and pairwise potential $D\left(x_{i}, y_{i}\right)(\mathrm{i}=\mathrm{j}, \mathrm{j}[\{1 \ldots$ $\mathrm{n}\})$. It is assumed $\mathrm{P}(\mathrm{y} / \mathrm{x})$ be the probability that can obtain labelling $\mathrm{y}$ of given data $\mathrm{x}$ and the parameter log-likelihood is defined as

$$
\begin{gathered}
-\log P\left(y / x_{i} ; \lambda\right)=\lambda \sum_{i \in v} S\left(x_{i}, y_{i}\right)+(1-\lambda) \sum_{(i, j) \in \varepsilon} D\left(y_{i}, y_{j}\right) \\
\text { IV. RESULT ANALYSIS }
\end{gathered}
$$

\section{RESULT ANALYSIS}

The simulation of the CRF model and the contour based shadow detection approach provides the result as shown in figure 4 .

The different performance parameters are used for the analysis of both the methods. The omitted error (EO) is produced by proper dark pixels recognized as non-shadow pixels. The committed error (EC) is produced by non-shadow pixels recognized like shade pixels. Since the committedomitted inaccuracies are not related and their results on the overall inaccuracy are the same in character, the total error (ET) is defined as their summation. The committed, omitted, and total errors are expressed as [14].

$$
E_{O}=\frac{F N}{T S} ; \quad E_{C}=\frac{F P}{T S} ; \quad E_{T}=E_{O}+E_{C}
$$

Where the number of true shadow pixels are denoted false negative (FN) which are identified as non-shadow pixels. The number of non-shadow pixels, identified as shadow pixels are denoted by False positive (FP). The total shadow (TS) denotes the entire number of true shadow pixels in the ideal shadow map.

Abhishek Mishra, Bharti Chaurasia and Yashwant Kurmi, " Comparative Analysis of Single Image Shadow Detection and Removal in Aerial Images," International Journal of Advanced Engineering and Management, vol. 2, no. 4, pp. 86-89, 2017.

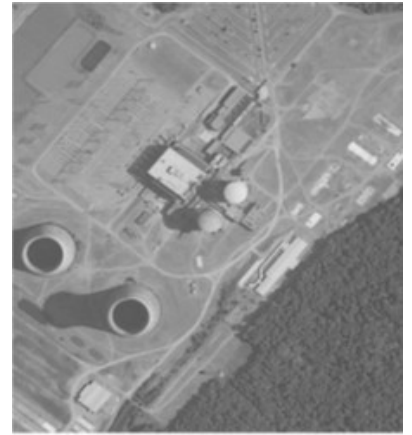

(a)

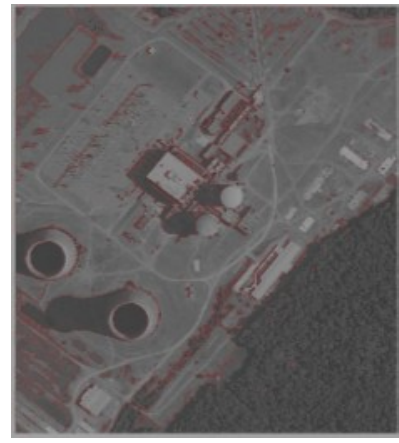

(c)

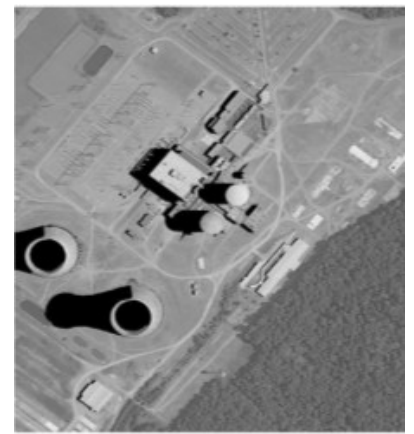

(b)

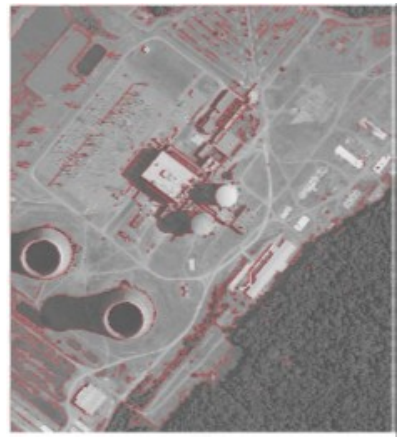

(d)
Fig.4 (a) Input satellite image used in the experiment. (b) The ground truth of the shadow for man-made buildings. (c) Geometric active contours based shadow detection results. (d) the CRF method based shadow detection results. Table 1 comparative analysis of the method 1 and method 2 for the images

\begin{tabular}{|l|c|c|c|}
\hline \multicolumn{1}{|c|}{ Method } & $\begin{array}{c}\text { Omitted } \\
\text { Error }\end{array}$ & $\begin{array}{c}\text { Committed } \\
\text { Error }\end{array}$ & $\begin{array}{l}\text { Total } \\
\text { Error }\end{array}$ \\
\hline $\begin{array}{l}\text { Contour based } \\
\text { shadow detection [1] }\end{array}$ & 6.35 & 10.84 & 17.29 \\
\hline $\begin{array}{l}\text { CRF based shadow } \\
\text { detection [2] }\end{array}$ & 4.86 & 8.49 & 13.35 \\
\hline
\end{tabular}

Table 1 shows the Contour based shadow detection method [1] and the conditional random field (CRF) based shadow detection method [2]. The total error for the contour detection 
method is 17.29 and for the CRF based shadow detection method has the value of total error is 13.35 . The comparative result is good for the CRF method as $22.78 \%$ in terms of total error.

\section{CONCLUSION}

Basically this paper compared the work done on the contour model based shadow detection algorithm with the random field model based shadow detection method. The contour model based on shadow recognition algorithm couture the usual form of the geometric active form like as the new model. After detection and segmentation of the shadow and the shaded portion in the figure the next step is calculated to find out the original report. Whereas the random field model based on shadow recognition algorithm uses a plane descriptor, to capture measured shaded surface deviation. The colour shaded portion incorporated into the situation casual field model to locate identical clarification pair and to acquire rational shaded area. The shadow removal method with local colour constancy computation uses less anisotropic diffusion to calculate the approximate the image pixel illumination in shadow region. The performance of the CRF method is $22.78 \%$ improved as evaluate to the contour based shade recognition method in terms of total error.

\section{REFERENCES}

[1] Elbakary, M. I., \& Iftekharuddin, K. M. (2014). Shadow detection of man-made buildings in high-resolution panchromatic satellite images. IEEE Transactions on Geoscience and Remote Sensing, 52(9), 5374-5386.

[2] Yuan, X., Ebner, M., \& Wang, Z. (2014). Single-image shadow detection and removal using local colour constancy computation. IET Image Processing, 9(2), 118-126.

[3] Zhu, J., Samuel, K. G., Masood, S. Z., \& Tappen, M. F. (2010, June). Learning to recognize shadows in monochromatic natural images. In Computer Vision and Pattern Recognition (CVPR), 2010 IEEE Conference on (pp. 223-230). IEEE.

[4] Lalonde, J. F., Efros, A. A., \& Narasimhan, S. G. (2009, September). Estimating natural illumination from a single outdoor image. In Computer Vision, 2009 IEEE 12th International Conference on (pp. 183-190). IEEE.

[5] Karsch, K., Hedau, V., Forsyth, D., \& Hoiem, D. (2011, December). Rendering synthetic objects into legacy photographs. In ACM Transactions on Graphics (TOG) (Vol. 30, No. 6, p. 157). ACM.

[6] Ghanbari, M., Majdi, M., \& Talouki, M. (2017). Video Inpainting Using a Contour-based Method in Presence of More than One Moving Objects. International Journal of Advanced Engineering and Management, 2(2), 37-44.

[7] Katre,R \& Dodkey, N.(2017)."Rain Streaks Removal in Image via Decomposition and Visibility Feature
Saturation.International Journal of Advanced Engineering and Management, 2(4), 82-85.

[8] Sharma, D., Kurmi, Y., \& Chaurasia, V. (2014). Formation of Super-Resolution Image: A Review. Int. Jour. of Emerging Tech. and Adv. Engg, 4(4), 218-221.

[9] Kurmi, Y., \& Chaurasia, V. (2015). An Image Fusion Approach based on Adaptive Fuzzy Logic Model with Local Level Processing. International Journal of Computer Applications, 124(1), 39-42

[10] Tiwari, S., Chauhan, K., \& Kurmi, Y. (2015). Shadow detection and compensation in aerial images using MATLAB. International Journal of Computer Applications, 119(20).

[11] Kurmi, Y., \& Chaurasia, V. (2014). Performance of Haze Removal Filter for Hazy and Noisy Images. Int. Jour. of Sci. Engg. and Tech, 3(4), 437-439.

[12] Patle, M. K., Chourasia, B. \& Kurmi. Y. (2016). "High Dynamic Range Image Analysis Through Various Tone Mapping Techniques. Int. Jour. of Comp. Appl., vol.153( 11),14-17

[13] Kumar, A., \& Chourasia, B. (2017). Image Dehazing (Defogging) by using Depth Estimation and Fusion with Guided Filter. International Journal of Computer Applications, 158(8).

[14] Liu, J., Fang, T., \& Li, D. (2011). Shadow detection in remotely sensed images based on self-adaptive feature selection. IEEE Transactions on Geoscience and Remote Sensing, 49(12), 5092-5103.

[15] Chan, T. F., \& Vese, L. A. (2001). Active contours without edges. IEEE Transactions on image processing, 10(2), 266277.

[16] Zhao, H. K., Chan, T., Merriman, B., \& Osher, S. (1996). A variational level set approach to multiphase motion. Journal of computational physics, 127(1), 179-195.

[17] Fredembach, C. \& Finlayson, G. (2005).Hamiltonian PathBased Shadow Removal. Proc. on 16th British Machine Vision Conf., Oxford, UK, 24(5), 603-619.

[18] Guo, R., Dai, Q., \& Hoiem, D. (2013). Paired regions for shadow detection and removal. IEEE transactions on pattern analysis and machine intelligence, 35(12), 2956-2967.

\footnotetext{
Abhishek Mishra

Dept. of ECE, Scope College of Engineering, Bhopal, India Email: setu17687@gmail.com

Bharti Chaurasia

Dept. of ECE, Scope College of Engineering, Bhopal, India Email: bharti.chourasia27@gmail.com

Yashwant Kurmi

Dept. of ECE, Maulana Azad National Institute of Technology Bhopal, India

Email:yashwantkurmi18@gmail.com
} 